\title{
HIV CRF07_BC is Independently Associated With Slower Disease Progression Than Subtype B in Chinese Patients: A Retrospective Observational Cohort Study
}

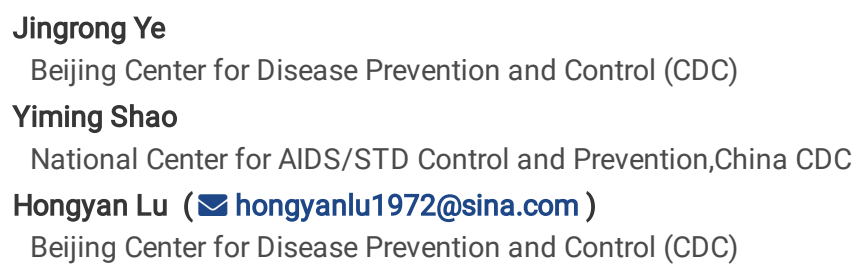

\section{Research Article}

Keywords: HIV, AIDS, disease progression, subtype, molecular epidemiology

Posted Date: August 23rd, 2021

DOI: https://doi.org/10.21203/rs.3.rs-809434/v1

License: (c) (1) This work is licensed under a Creative Commons Attribution 4.0 International License. Read Full License 


\section{Abstract}

HIV subtypes convey important epidemiological information and possibly influence the rate of disease progression. In this study, HIV disease progression in patients infected with CRF01_AE, CRF07_BC, and subtype B was compared in the largest HIV molecular epidemiology study ever done in China. A national data set of HIV pol sequences was assembled by pooling sequences from public databases and the Beijing HIV laboratory network. Logistic regression was used to assess factors associated with the risk of AIDS at diagnosis ([AIDSAD], defined as a CD4 count <200 cells/ $\mu \mathrm{L}$ ) in patients with HIV subtype B, CRF01_AE, and CRF07_BC. Of the 20,663 sequences, 9,156 (44.3\%) were CRF01_AE. CRF07_BC was responsible for $28.3 \%$ of infections, followed by $B(13.9 \%)$. In multivariable analysis, the risk of AIDSAD differed significantly according to HIV subtype (OR for CRF07_BC vs. B: $0.46,95 \% \mathrm{Cl} 0.39-0.53$ ), age (OR for $\geq 65$ years vs. $<18$ years: $4.395 \% \mathrm{Cl} 1.81-11.8$ ), and transmission risk groups (OR for man who have sex with man vs. heterosexual: $0.6795 \% \mathrm{Cl} 0.6-0.75)$. These findings suggest that HIV diversity in China is constantly evolving and gaining complexity. CRF07_BC is less pathogenic than subtype B, while CRF01_AE is as pathogenic as B.

\section{Introduction}

China has a slowly increasing HIV epidemic, with 64,170, 71,204, and 63,154 new cases in 2018, 2019, and 2020, and 818,360 individuals living with HIV at the end of 2020. ${ }^{1}$ During the first two decades of the epidemic (1985-2005), most HIV cases were concentrated in the injection drug users (IDU [44.2\%]) and former blood donors (29.6\%), but since 2006, there has been a clear expansion of the HIV cases in heterosexuals and men who have sex with men (MSM). In 2019, heterosexuals and MSM accounted for $73.8 \%$ and $23.3 \%$ of new diagnoses, respectively, with IDU accounting for $3.4 \%{ }^{2}$ Understanding the increase in HIV diversity within China is not only of epidemiological interest but also has far-reaching clinical implications. ${ }^{3-9}$

One of the fascinating findings concerning the HIV subtype in China is the belief that CRF01_AE progresses faster than CRF07_BC. ${ }^{10-14}$ However, these studies were limited by small sample sizes and failed to adjust for important confounding factors. Worldwide, consistent findings regarding the rates of disease progression among different HIV subtypes in descending order are subtype $C>D>C R F 01 \_A E>G>A .{ }^{15-26} A$ lthough subtype $B$ is the most studied because of its predominance in North America and Europe, it is absent in this comparison chain.

When comparing subtype B with non-B strains using non-B as comparator, it is assumed that all subtypes except for B progress equally, which is obviously not the case. To date, no previous studies have been sufficiently large to directly compare subtype B with other single subtypes. ${ }^{15-26}$ The latest national HIV epidemiology study in China was conducted in 2006 and was published in $2012 .{ }^{4}$ Fourteen years have passed, and the China's epidemic has changed. In this study, HIV disease progression was compared between patients infected with subtype B, CRF01_AE, and CRF07_BC in the largest HIV molecular epidemiology study ever conducted in China.

\section{Methods}

Study population and design

The study population consisted of two separate populations of HIV-infected individuals. The first group comprised all patients with the HIV transmitted drug resistance (TDR) genotype, performed between 2001 and 2020 at the Beijing HIV laboratory network (BHLN). BHLN is a national collaboration engaged in surveillance of HIV TDR in China. ${ }^{29,30}$ These methods have been previously described. Briefly, approximately $40 \%$ of the samples from all individuals newly diagnosed with HIV infection by BHLN between 2001 and 2020 were randomly selected. ${ }^{29,30}$ The BHLN takes part in maintaining the national HIV epidemiology database, which tracks everyone who receives a diagnosis of HIV infection in China and records the baseline CD4 count of all individuals with newly diagnosed HIV infection. The baseline CD4 count was the value from their CD4 count closest to the date on which their HIV infection was confirmed by western blot within one year. Baseline demographic data on sex, age, ethnicity, Hukou province, and the transmission risk group were retrieved from this database.

The second group included publicly available sequences from the Los Alamos HIV sequence database. All the pol sequences sampled in China with known sampling provinces, sampling years, and transmission risk groups available in the database were downloaded (data available as of December 1 , 2019).

Phylogenetic analysis

Sequences were aligned using the BioEdit tool and the alignment was manually corrected according to the encoded reading frame. Duplicate sequences were discarded. If several sequences from the same patient were available in the database, only the oldest was retained. Long branch sequences were re-confirmed for their genotype, and those that were miscatalogued were eliminated from the study.

A maximum likelihood phylogenetic tree was reconstructed with the merged dataset using the GTR + CAT nucleotide substitution model in Fast Tree 2.1. ${ }^{36}$ The HIV subtype was inferred by automated subtyping using context-based modeling for expeditious typing (COMET), ${ }^{37}$ followed by phylogenetic analysis. Each sequence was assigned to one of eight subtypes, one of 102 circulating recombinant forms (CRF), or "unassigned." An "unassigned" sequence was deemed as a possible unique recombinant forms (URF). ${ }^{6}$

Cohort of natural disease progression 
The BHLN may also be used as a cohort to study natural disease progression of HIV in China. The starting point of the study was set as the onset of the infection and the outcome was laboratory acquired immunodeficiency syndrome ([AIDS], defined as a CD 4 count $<200$ cells/ $\mu \mathrm{L}$ ) at diagnosis (AIDSAD). The follow-up time was the duration between the starting point and the outcome.

As the seroconversion time for most of the participants was unavailable, the follow-up time was unmeasurable. To solve this problem, the follow-up time was treated as a matching variable in cohort analysis, as we hypothesized that the distribution of the follow-up time was well matched within the same transmission risk group and roughly matched the study population as a whole. Two sensitivity analysis were performed by limiting the comparison of subtypes in heterosexuals and MSM.

\section{Statistical analysis}

For geographic location, participants were grouped into 31 provinces according to the Hukou. Hukou is a basic household registration system in China; this system officially identifies a person as a resident of an area and includes identifying information such as name, parents, spouse, and date of birth. These provinces were further divided into six regions according to their proximity and socio-economic status, in line with guidelines from the National Bureau of Statistics of China: north, northeast, east, central-south, southwest, and northwest.

Six sampling phases were established:1994-2005, 2006-2008, 2009-2011, 2012-2014, 2015-2017, and 2018-2020. The earliest (1994-2005) phase encompassed more years to account for the relatively fewer data available in these years. The prevalence of subtype by sex, age, ethnicity, transmission risk group, Hukou province, and region was calculated and the subtype distribution trends over the six sampling phases were examined. Categorical data were compared using the X2 test and continuous data were compared using one-way analysis of variance, wherever appropriate.

Potential risk factors for acquiring AIDSAD were analyzed using logistic regression. Biologically plausible interactions were assessed in the multivariable model. Variables included sex, age $(<18,18-24,25-44,45-64$, and $\geq 65$ years), ethnicity, region, subtype, transmission risk group, and sampling phase. In the model, a binary response was included, indicating the acquisition AIDSAD from each patient as an outcome. All variables were analyzed separately and the associated variables $(P<0.1)$ with their outcomes were entered into the multivariable model.

Results are expressed as odds ratios (OR) with 95\% confidence intervals (Cl) and two-sided $P$ values, where $P<0.05$ was considered statistically significant. All analyses were performed using R software (version 3.6.1; R Foundation, Vienna, Austria) and a listwise deletion was used to handle the missing data.

Ethical issues

All analyses were performed on de-identified datasets to protect participants' anonymity. The research ethics committee at the Beijing center for disease prevention and control approved this study, and all the methods in this study were performed in accordance with the approved guidelines. By law, consent was not required as these data were collected and analyzed in the course of routine public health surveillance.

\section{Results}

Study Population

HIV polsequences generated from 13,230 patient specimens submitted by the BHLN for TDR genotyping between 2001 and 2020 were analyzed. A total of 7,433 pol sequences sampled in China were retrieved, of which the province of origin, transmission risk group and sampling year were available from the Los Alamos HIV sequence database. In all, 20,663 aligned HIV pol sequences were used in this analysis, each representing a distinct HIV-positive individual (Fig. 1). These data were collected between 1994 and 2020 from 31 provinces of China.

Most participants were men (94.2\%) of Han ethnicity (93.1\%). The median age was 32 years (interquartile range [IQR] 26-42). Where available, the overall median baseline CD4 count was 338cells/ $\mu \mathrm{L}$ (IQR 208-475). The transmission risk group was predominantly MSM (66.6\%), followed by heterosexual (23.3\%) and IDU (7.7\%) (Table 1). 
Table 1

Baseline characteristic by sampling phase.

\begin{tabular}{|c|c|c|c|c|c|c|c|c|}
\hline & 1994-2005 & $2006-2008$ & 2009-2011 & 2012-2014 & 2015-2017 & 2018-2020 & Total & $\begin{array}{l}P \\
\text { value }\end{array}$ \\
\hline \multicolumn{9}{|l|}{ Sex } \\
\hline Men & 169(70.71) & 1162(86.07) & 4162(95.68) & $4714(96.5)$ & $3651(95.48)$ & 3293(92.5) & 17151(94.19) & $<0.05$ \\
\hline Women & 70(29.29) & 188(13.93) & 188(4.32) & 171(3.5) & $173(4.52)$ & $267(7.5)$ & 1057(5.81) & \\
\hline Age(years) ${ }^{a}$ & $32(26-38)$ & $31(25-38)$ & $31(26-39)$ & $31(26-40)$ & $31(26-44)$ & $33(27-45)$ & $32(26-42)$ & NS \\
\hline \multicolumn{9}{|l|}{ Ethnicity } \\
\hline Han & 173(77.58) & 756(76.06) & $1790(91.42)$ & 2505(93.54) & $3648(95.57)$ & $3442(96.69)$ & 12314(93.08) & $<0.05$ \\
\hline Uyghur & $40(17.94)$ & 139(13.98) & $54(2.76)$ & $29(1.08)$ & $27(0.71)$ & $6(0.17)$ & $295(2.23)$ & \\
\hline Yi & $2(0.9)$ & $35(3.52)$ & $38(1.94)$ & $34(1.27)$ & $20(0.52)$ & $12(0.34)$ & 141(1.07) & \\
\hline Other & $8(3.59)$ & $64(6.44)$ & $76(3.88)$ & $110(4.11)$ & $122(3.2)$ & $100(2.81)$ & $480(3.63)$ & \\
\hline $\begin{array}{l}\text { CD4 count(cells per } \\
\mu \mathrm{l})^{\mathrm{b}}\end{array}$ & $\begin{array}{l}299(181- \\
402)\end{array}$ & $\begin{array}{l}330(209- \\
460)\end{array}$ & $\begin{array}{l}345(202- \\
455)\end{array}$ & $\begin{array}{l}365(235- \\
510)\end{array}$ & $\begin{array}{l}326(204- \\
462)\end{array}$ & $\begin{array}{l}323(200- \\
470)\end{array}$ & $\begin{array}{l}338(208- \\
475)\end{array}$ & NS \\
\hline \multicolumn{9}{|l|}{$\begin{array}{l}\text { Transmission risk } \\
\text { groups }\end{array}$} \\
\hline Heterosexual & 149(29.22) & $616(31.4)$ & 1045(19.27) & 911(17.34) & 837(21.96) & $1227(34.51)$ & 4785(23.32) & $<0.05$ \\
\hline MSM & $50(9.8)$ & 742(37.82) & $3581(66.03)$ & $4120(78.42)$ & 2867(75.23) & $2295(64.54)$ & $13655(66.56)$ & \\
\hline Injecting drug user & 111(21.76) & $418(21.3)$ & 712(13.13) & 203(3.86) & $107(2.81)$ & $34(0.96)$ & 1585(7.73) & \\
\hline Blood transfusion & 190(37.25) & 176(8.97) & $79(1.46)$ & $16(0.3)$ & $0(0)$ & $0(0)$ & $461(2.25)$ & \\
\hline Mother to Child & 10(1.96) & $10(0.51)$ & $6(0.11)$ & $4(0.08)$ & $0(0)$ & $0(0)$ & $30(0.15)$ & \\
\hline Region & y199405 & y200608 & y200911 & y201214 & y201517 & y201820 & & $<0.05$ \\
\hline North & 104(19.85) & $554(27.62)$ & $1250(22.97)$ & $1721(32.73)$ & 1942(50.78) & 1719(48.41) & $7290(35.38)$ & \\
\hline North-east & 78(14.89) & $104(5.18)$ & $513(9.43)$ & $393(7.47)$ & $525(13.73)$ & $561(15.8)$ & $2174(10.55)$ & \\
\hline East & 79(15.08) & 295(14.71) & $1155(21.22)$ & $1386(26.36)$ & $461(12.06)$ & $437(12.31)$ & $3813(18.51)$ & \\
\hline Central-south & 148(28.24) & 707(35.24) & 1196(21.98) & 1086(20.65) & $464(12.13)$ & 438(12.33) & 4039(19.6) & \\
\hline South-west & 71(13.55) & 162(8.08) & $1180(21.68)$ & $517(9.83)$ & $234(6.12)$ & $216(6.08)$ & $2380(11.55)$ & \\
\hline North-west & $44(8.4)$ & 184(9.17) & $148(2.72)$ & $155(2.95)$ & 198(5.18) & $180(5.07)$ & $909(4.41)$ & \\
\hline \multicolumn{9}{|l|}{ Subtype } \\
\hline B & $250(47.71)$ & $602(29.86)$ & 843(15.39) & $570(10.84)$ & $377(9.86)$ & $235(6.6)$ & 2877(13.92) & $<0.05$ \\
\hline$C$ & $22(4.2)$ & $31(1.54)$ & $105(1.92)$ & $55(1.05)$ & $27(0.71)$ & $19(0.53)$ & $259(1.25)$ & \\
\hline 01_AE & 103(19.66) & 679(33.68) & $2423(44.23)$ & $2586(49.16)$ & 1797(46.98) & 1568(44.04) & $9156(44.31)$ & \\
\hline 07_BC & $89(16.98)$ & $486(24.11)$ & 1639(29.92) & $1424(27.07)$ & 1108(28.97) & 1096(30.79) & $5842(28.27)$ & \\
\hline 08_BC & $26(4.96)$ & $103(5.11)$ & $70(1.28)$ & $93(1.77)$ & $33(0.86)$ & $32(0.9)$ & $357(1.73)$ & \\
\hline 55_01B & $1(0.19)$ & 28(1.39) & 108(1.97) & $149(2.83)$ & $66(1.73)$ & $99(2.78)$ & $451(2.18)$ & \\
\hline Minor & $14(2.67)$ & $38(1.88)$ & $115(2.1)$ & $94(1.79)$ & $101(2.64)$ & $137(3.85)$ & $499(2.41)$ & \\
\hline URF & 19(3.63) & $49(2.43)$ & 175(3.19) & $289(5.49)$ & $316(8.26)$ & $374(10.51)$ & $1222(5.91)$ & \\
\hline \multicolumn{9}{|c|}{ Data are $\mathrm{n}(\%)$, or median (IQR). } \\
\hline \multicolumn{9}{|c|}{ MSM, Man who have sex with man. } \\
\hline \multicolumn{9}{|c|}{ URF, unique recombinant form. } \\
\hline \multicolumn{9}{|c|}{ North, Beijing, Tianjin, Hebei, Shanxi, and Inner Mongolia; } \\
\hline \multicolumn{9}{|c|}{ North-east, Liaoning, Jilin, and Heilongjiang; } \\
\hline
\end{tabular}




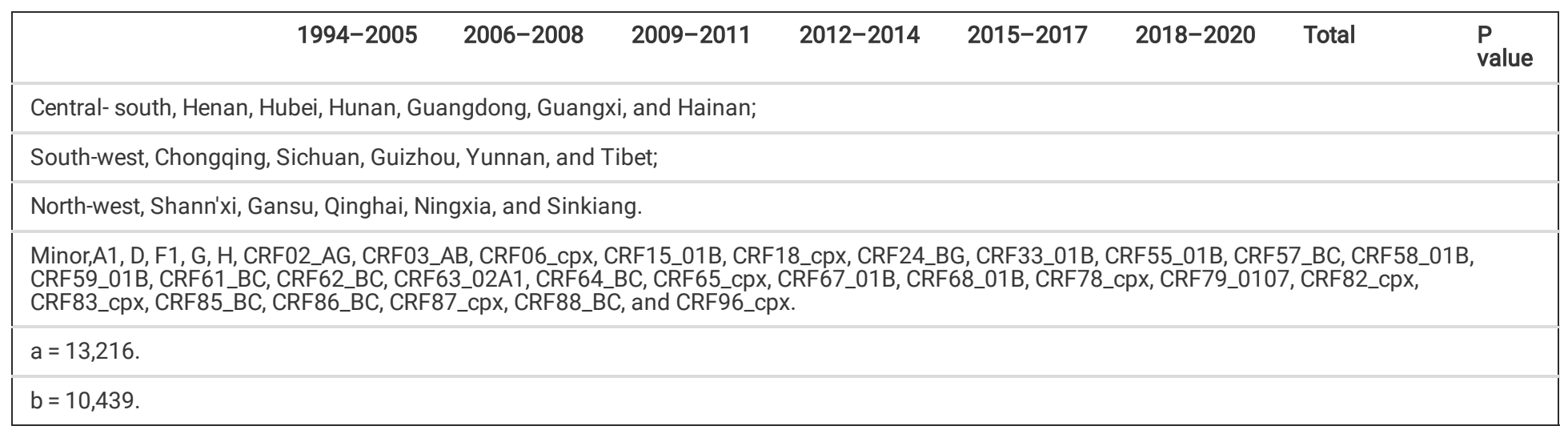

HIV subtype distribution

A total of 38 HIV subtypes and CRF were identified in the study. CRF01_AE, CRF07_BC, subtype B, URF, CRF55_01B, CRF08_BC, and subtype C (seven common subtypes) were the seven predominant HIV subtypes circulating in China, accounting for $44.3 \%, 28.3 \%, 13.9 \%, 5.9 \%, 2.2 \%, 1.7 \%$, and $1.3 \%$ of all infections, respectively (Table 2). 
Table 2

Subtype assignment by selected characteristics

\begin{tabular}{|c|c|c|c|c|c|c|c|c|c|}
\hline & B & C & 01_AE & 07_BC & 08_BC & 55_01B & minor & URF & total \\
\hline \multicolumn{10}{|l|}{ Sex } \\
\hline Men & 2217(12.93) & $79(0.46)$ & 8201(47.82) & 4762(27.77) & $104(0.61)$ & $425(2.48)$ & $361(2.1)$ & $1002(5.84)$ & $17151(100)$ \\
\hline Women & $266(25.17)$ & $33(3.12)$ & 284(26.87) & $323(30.56)$ & $55(5.2)$ & $8(0.76)$ & $53(5.01)$ & $35(3.31)$ & 1057(100) \\
\hline \multicolumn{10}{|l|}{$\begin{array}{l}\text { Age at } \\
\text { diagnosis(years) }\end{array}$} \\
\hline$<18$ & $33(36.67)$ & $3(3.33)$ & $20(22.22)$ & $26(28.89)$ & $1(1.11)$ & $1(1.11)$ & $3(3.33)$ & 3(3.33) & $90(100)$ \\
\hline $18-24$ & 222(10.63) & $10(0.48)$ & $987(47.27)$ & $661(31.66)$ & $12(0.57)$ & $31(1.48)$ & $40(1.92)$ & 125(5.99) & 2088(100) \\
\hline $25-44$ & $1246(15.02)$ & $50(0.6)$ & $3866(46.59)$ & $2225(26.81)$ & $87(1.05)$ & $117(1.41)$ & $167(2.01)$ & $540(6.51)$ & 8298(100) \\
\hline $45-64$ & 391(16.24) & $31(1.29)$ & $971(40.32)$ & 635(26.37) & $36(1.5)$ & $50(2.08)$ & $107(4.44)$ & 187(7.77) & $2408(100)$ \\
\hline$\geq 65$ & $44(13.25)$ & $5(1.51)$ & 111(33.43) & 114(34.34) & $11(3.31)$ & $5(1.51)$ & 13(3.92) & $29(8.73)$ & $332(100)$ \\
\hline \multicolumn{10}{|l|}{ Ethnicity } \\
\hline Han & 1854(15.06) & $89(0.72)$ & $5734(46.56)$ & $3135(25.46)$ & $130(1.06)$ & 196(1.59) & $320(2.6)$ & $856(6.95)$ & $12314(100)$ \\
\hline Uyghur & $8(2.71)$ & $1(0.34)$ & $5(1.69)$ & 277(93.9) & $0(0)$ & $0(0)$ & $2(0.68)$ & $2(0.68)$ & 295(100) \\
\hline Yi & $3(2.13)$ & $2(1.42)$ & $5(3.55)$ & 121(85.82) & $4(2.84)$ & $0(0)$ & $0(0)$ & $6(4.26)$ & $141(100)$ \\
\hline Others & $73(15.21)$ & $7(1.46)$ & 218(45.42) & $132(27.5)$ & $13(2.71)$ & $8(1.67)$ & $8(1.67)$ & $21(4.38)$ & $480(100)$ \\
\hline $\begin{array}{l}\text { CD } 4 \text { count(cells } \\
\text { per } \mu \mathrm{l})^{b}\end{array}$ & $\begin{array}{l}301(160- \\
435)\end{array}$ & $\begin{array}{l}333(173- \\
514)\end{array}$ & $\begin{array}{l}328(191- \\
464)\end{array}$ & $\begin{array}{l}366(253- \\
501)\end{array}$ & $\begin{array}{l}290(164- \\
426)\end{array}$ & $\begin{array}{l}327(205- \\
481)\end{array}$ & $\begin{array}{l}335(223- \\
487)\end{array}$ & $\begin{array}{l}356(225- \\
493)\end{array}$ & $\begin{array}{l}338(208- \\
475)\end{array}$ \\
\hline \multicolumn{10}{|l|}{$\begin{array}{l}\text { Transmission risk } \\
\text { groups }\end{array}$} \\
\hline Heterosexual & $735(15.36)$ & $133(2.78)$ & 2041(42.65) & $1088(22.74)$ & 207(4.33) & $76(1.59)$ & 190(3.97) & $315(6.58)$ & $4785(100)$ \\
\hline MSM & $1640(12.01)$ & $39(0.29)$ & 6856(50.21) & $3672(26.89)$ & $39(0.29)$ & $372(2.72)$ & 254(1.86) & 783(5.73) & $13655(100)$ \\
\hline $\begin{array}{l}\text { Injecting drug } \\
\text { user }\end{array}$ & 63(3.97) & $80(5.05)$ & 161(10.16) & 1018(64.23) & $105(6.62)$ & $3(0.19)$ & $36(2.27)$ & $119(7.51)$ & 1585(100) \\
\hline $\begin{array}{l}\text { Blood } \\
\text { transfusion }\end{array}$ & $378(82)$ & $0(0)$ & 41(8.89) & $31(6.72)$ & $3(0.65)$ & $0(0)$ & $7(1.52)$ & $1(0.22)$ & $461(100)$ \\
\hline Mother to Child & $15(50)$ & $1(3.33)$ & $6(20)$ & $3(10)$ & $2(6.67)$ & $0(0)$ & $3(10)$ & $0(0)$ & $30(100)$ \\
\hline \multicolumn{10}{|l|}{ Region } \\
\hline North & 1198(16.43) & $57(0.78)$ & $3460(47.46)$ & 1738(23.84) & $49(0.67)$ & 101(1.39) & $201(2.76)$ & $486(6.67)$ & $7290(100)$ \\
\hline North-east & 281(12.93) & $13(0.6)$ & 1211(55.7) & $460(21.16)$ & $15(0.69)$ & $21(0.97)$ & $52(2.39)$ & $121(5.57)$ & 2174(100) \\
\hline East & $449(11.78)$ & $17(0.45)$ & 2108(55.28) & $887(23.26)$ & $52(1.36)$ & $66(1.73)$ & $88(2.31)$ & 146(3.83) & $3813(100)$ \\
\hline Central-south & 686(16.98) & $19(0.47)$ & 1592(39.42) & $1153(28.55)$ & $97(2.4)$ & $243(6.02)$ & $52(1.29)$ & 197(4.88) & $4039(100)$ \\
\hline South-west & $151(6.34)$ & $149(6.26)$ & $480(20.17)$ & $1124(47.23)$ & $137(5.76)$ & $16(0.67)$ & $91(3.82)$ & 232(9.75) & $2380(100)$ \\
\hline North-west & $94(10.34)$ & $2(0.22)$ & 279(30.69) & $472(51.93)$ & $6(0.66)$ & $4(0.44)$ & $13(1.43)$ & $39(4.29)$ & $909(100)$ \\
\hline \multicolumn{10}{|c|}{ Data are $\mathrm{n}(\%)$, or median (IQR). } \\
\hline \multicolumn{10}{|c|}{ MSM, Man who have sex with man. } \\
\hline \multicolumn{10}{|c|}{ URF, unique recombinant form. } \\
\hline \multicolumn{10}{|c|}{ North, Beijing, Tianjin, Hebei, Shanxi, and Inner Mongolia; } \\
\hline \multicolumn{10}{|c|}{ North-east, Liaoning, Jilin, and Heilongjiang; } \\
\hline \multicolumn{10}{|c|}{ East, Shanghai, Jiangsu, Zhejiang, Anhui, Fujian, Jiangxi, and Shandong; } \\
\hline \multicolumn{10}{|c|}{ Central- south, Henan, Hubei, Hunan, Guangdong, Guangxi, and Hainan; } \\
\hline South-west, Chor & $\mathrm{g}$, Sichuan, $\mathrm{C}$ & zhou, Yunn & , and Tibet; & & & & & & \\
\hline
\end{tabular}




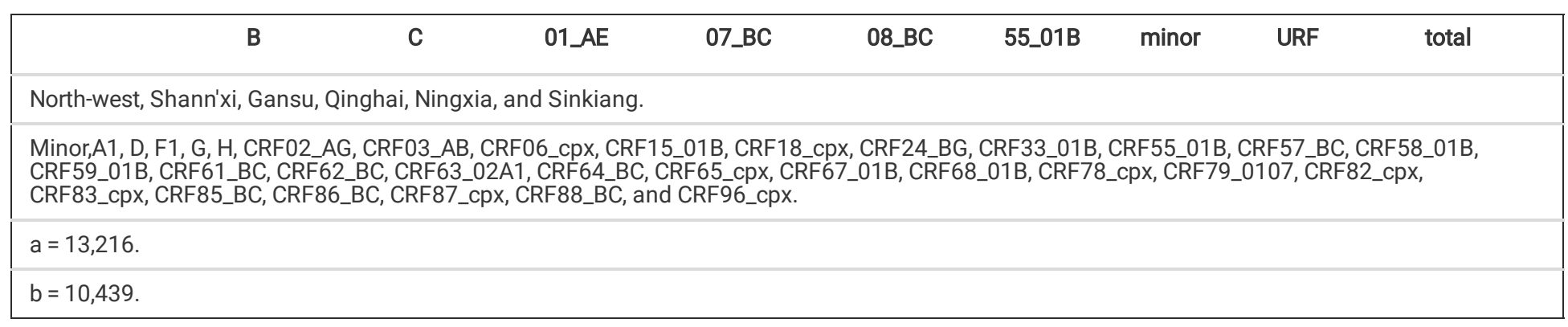

Additional clades including subtypes A1, D, F1, G,H, CRF02_AG, CRF03_AB, CRF06_cpx,CRF15_01B, CRF18_cpx, CRF24_BG, CRF33_01B, CRF55_01B,CRF57_BC, CRF58_01B, CRF59_01B, CRF61_BC, CRF62_BC, CRF63_02A1, CRF64_BC, CRF65_cpx,CRF67_01B, CRF68_01B, CRF78_cpx, CRF79_0107, CRF82_cpx, CRF83_cpx, CRF85_BC,CRF86_BC, CRF87_cpx, CRF88_BC, and CRF96_cpx (minor subtypes) were present in less than 1.0\% of individuals. Of the subtypes, the combined prevalence of CRF01_AE, CRF07_BC, and subtype B (three major subtypes) remained steady at just over 85\%, while the foreign subtypes(subtypes that originated and circulated mainly in foreign countries, including subtypes A1, D, F1, G, H, CRF 02_AG, CRF03_AB, CRF06_cpx, CRF15_01B, CRF18_cpx,CRF24_BG,CRF33_01B, CRF58_01B, CRF63_02A1, CRF_82cpx, and CRF_83cpx) constituted only 3\% of all the subtypes.

The prevalence of subtypes varied significantly according to sex, age, ethnicity, transmission risk group, and region. Table 2 shows the subtype diversity within the demographic subgroups. Women had a greater prevalence of infection with subtype B and a lower prevalence of infection with CRF01_AE and URF. Older individuals ( $\geq 65$ years) tended to have lower prevalence of infection with CRF01_AE. Genotypic diversity was the greatest among heterosexuals, in which 35 HIV genotype categories were detected, the most prevalent being CRF01_AE. MSM included 21 subtypes, of which CRF01_AE was the most prevalent. IDU most likely had CRF07_BC. Individuals of Uyghur and Yi ethnicity predominantly, though not exclusively, were infected with the CRF07_BC virus. Figure 2. illustrates the regional distributions of the seven common subtypes HIV strains. CRF07_BC was more prevalent in the southwest and northwest region. In the other four regions, the subtype with the highest prevalence was CRF01_AE. Of note, a significantly high prevalence of URF was detected in the southwest region $(9.6 \%)$.

HIV subtype temporal trends

Table 1 presents the temporal trends for the seven common subtypes. The prevalence of CRF01_AE increased from 19.7-49.2\% between the phase 1994-2005 and 2012-2014 and remained high. A similar trend was observed for CRF07_BC. Interestingly, the prevalence of subtype B decreased from $47.7 \%$ in phase $1994-2005$ to $6.6 \%$ in 2018-2020. Time trends were also examined by sex, age, ethnicity, transmission risk group, and region (table S1$5)$.

\section{Phylogenetic analysis}

Phylogenetic analysis revealed that the sequences from both sources were intermixed, suggesting that both sampling frames were drawn from the same overall population(Fig S1-3). Three, seven, and four distinct clusters were identified within subtype B, CRF01_AE, and CRF07_BC, including 14,578 individuals ( $81.6 \%$ of all patients infected with the three major subtypes). The clusters have been named based on a previous numbering system ${ }^{27}$ and with the addition of new clusters in the current study. The cluster size ranged from 175-2,964 individuals. Most clusters were MSM dominated (10 of 14). Table S6 presents the detailed characteristics of these clusters.

\section{CRF07_BC progressed slower than subtype B}

To study the effect of subtypes on the disease progression, the analysis was limited to the individuals with available CD4 counts. CD4 counts of the seven common subtypes were compared overall and by sampling phase. The CD4 count of CRF07_BC was always significantly higher than that of subtype B and CRF01_AE $(P<0.01)$ (Fig. 3). Therefore, we empirically hypothesized that disease progression in these subtypes could be different. Table 3 reports the association between patient characteristics and AIDSAD. 
Table 3

Demographic and clinical factors associated with AIDSAD

\begin{tabular}{|c|c|c|c|c|c|c|}
\hline & $\begin{array}{l}\text { Number of } \\
\text { sequences }\end{array}$ & $\begin{array}{l}\text { AIDSAD } \\
\text { prevalence }^{a}\end{array}$ & $\begin{array}{l}\text { Univariable } \\
\text { analysis }^{\text {b }}\end{array}$ & & $\begin{array}{l}\text { Multivariable } \\
\text { analysis }^{c}\end{array}$ & \\
\hline & & & OR $(95 \% \mathrm{Cl})$ & $\begin{array}{l}\mathrm{P} \\
\text { value }\end{array}$ & OR (95\% Cl) & $P$ value \\
\hline \multicolumn{7}{|l|}{ Sex } \\
\hline Men & 9681 & $2210(22.83)$ & Reference & & & \\
\hline Women & 758 & $210(27.7)$ & $1.29(1.1-1.53)$ & 0.002 & $0.86(0.71-1.04)$ & 0.12 \\
\hline \multicolumn{7}{|c|}{$\begin{array}{l}\text { Age at diagnosis(years) } \\
\text { group }\end{array}$} \\
\hline$<18$ & 48 & 10(20.83) & Reference & & & \\
\hline $18-24$ & 1625 & 207(12.74) & $0.55(0.12-1.19)$ & 0.1 & $1.11(0.48-2.94)$ & 0.82 \\
\hline $25-44$ & 6559 & $1463(22.31)$ & $1.09(0.28-2.32)$ & 0.81 & $2.05(0.9-5.4)$ & 0.11 \\
\hline $45-64$ & 1962 & 648(33.03) & $1.87(0.56-4)$ & 0.08 & $3.36(1.47-8.87)$ & 0.007 \\
\hline$\geq 65$ & 240 & $91(37.92)$ & $2.32(1.14-5.13)$ & 0.03 & $4.32(1.81-11.75)$ & 0.002 \\
\hline \multicolumn{7}{|l|}{ Ethnicity } \\
\hline Han & 9879 & 2310(23.38) & Reference & & & \\
\hline Uyghur & 97 & $8(8.25)$ & $0.29(0.13-0.57)$ & $<.001$ & $0.49(0.21-1.02)$ & 0.08 \\
\hline Yi & 90 & $7(7.78)$ & $0.28(0.12-0.56)$ & 0.001 & $0.43(0.17-0.9)$ & 0.04 \\
\hline Other & 373 & $95(25.47)$ & $1.12(0.88-1.41)$ & 0.35 & $1.18(0.92-1.5)$ & 0.19 \\
\hline \multicolumn{7}{|c|}{ Transmission risk group } \\
\hline Heterosexual & 2855 & $841(29.46)$ & Reference & & & \\
\hline MSM & 7086 & 1454(20.52) & $0.62(0.56-0.68)$ & $<.001$ & $0.67(0.6-0.75)$ & $<0.001$ \\
\hline Injecting drug user & 312 & $51(16.35)$ & $0.47(0.34-0.63)$ & $<.001$ & $0.85(0.59-1.2)$ & 0.36 \\
\hline Blood transfusion & 112 & $51(45.54)$ & $2(1.36-2.93)$ & $<.001$ & $1.86(1.24-2.79)$ & 0.003 \\
\hline Mother to child & 10 & $4(40)$ & $1.6(0.41-5.6)$ & 0.47 & $3.36(0.7-15.3)$ & 0.12 \\
\hline \multicolumn{7}{|l|}{ Subtype } \\
\hline$B$ & 1508 & 452(29.97) & Reference & & & \\
\hline C & 72 & $20(27.78)$ & $0.9(0.51-1.5)$ & 0.69 & $0.82(0.47-1.39)$ & 0.48 \\
\hline 01_AE & 4819 & $1259(26.13)$ & $0.83(0.73-0.94)$ & 0.003 & $0.94(0.82-1.07)$ & 0.35 \\
\hline 07_BC & 2815 & 421(14.96) & $0.41(0.35-0.48)$ & $<0.001$ & $0.46(0.39-0.53)$ & $<0.001$ \\
\hline 08_BC & 95 & $29(30.53)$ & $1.03(0.65-1.59)$ & 0.91 & $0.83(0.51-1.3)$ & 0.42 \\
\hline 55_01B & 169 & $41(24.26)$ & $0.75(0.51-1.07)$ & 0.12 & $0.78(0.53-1.13)$ & 0.2 \\
\hline URF & 708 & 142(20.06) & $0.59(0.47-0.72)$ & $<0.001$ & $0.62(0.5-0.78)$ & $<0.001$ \\
\hline Minor & 253 & $56(22.13)$ & $0.66(0.48-0.91)$ & 0.01 & $0.63(0.45-0.86)$ & 0.005 \\
\hline \multicolumn{7}{|l|}{ Region } \\
\hline North & 5061 & 1191(23.53) & Reference & & & \\
\hline North-east & 1486 & $338(22.75)$ & $0.96(0.83-1.1)$ & 0.53 & & \\
\hline East & 1251 & 293(23.42) & $0.99(0.86-1.15)$ & 0.93 & & \\
\hline Central-south & 1324 & 313(23.64) & $1(0.87-1.16)$ & 0.93 & & \\
\hline
\end{tabular}




\begin{tabular}{|c|c|c|c|c|}
\hline & $\begin{array}{l}\text { Number of } \\
\text { sequences }\end{array}$ & $\begin{array}{l}\text { AIDSAD } \\
\text { prevalence }^{a}\end{array}$ & $\begin{array}{l}\text { Univariable } \\
\text { analysis }^{\text {b }}\end{array}$ & $\begin{array}{l}\text { Multivariable } \\
\text { analysis }^{c}\end{array}$ \\
\hline South-west & 752 & $168(22.34)$ & $0.93(0.78-1.12)$ & 0.47 \\
\hline North-west & 542 & $110(20.3)$ & $0.83(0.66-1.03)$ & 0.09 \\
\hline \multicolumn{5}{|c|}{ Sampling Phase } \\
\hline 1994-2005 & 71 & $20(28.17)$ & Reference & \\
\hline $2006-2008$ & 574 & 133(23.17) & $0.77(0.45-1.36)$ & 0.35 \\
\hline 2009-2011 & 1537 & $372(24.2)$ & $0.81(0.49-1.41)$ & 0.45 \\
\hline 2012-2014 & 2562 & $516(20.14)$ & $0.64(0.39-1.11)$ & 0.1 \\
\hline 2015-2017 & 3101 & $735(23.7)$ & $0.79(0.48-1.37)$ & 0.38 \\
\hline $2018-2020$ & 2594 & $644(24.83)$ & $0.84(0.51-1.45)$ & 0.52 \\
\hline \multicolumn{5}{|c|}{ AIDSAD, AIDS at diagnose; } \\
\hline \multicolumn{5}{|c|}{ OR, odds ratio; } \\
\hline \multicolumn{5}{|c|}{ MSM, Man who have sex with man. } \\
\hline \multicolumn{5}{|c|}{ URF, unique recombinant form. } \\
\hline \multicolumn{5}{|c|}{ North, Beijing, Tianjin, Hebei, Shanxi, and Inner Mongolia; } \\
\hline \multicolumn{5}{|c|}{ North-east, Liaoning, Jilin, and Heilongjiang; } \\
\hline \multicolumn{5}{|c|}{ East, Shanghai, Jiangsu, Zhejiang, Anhui, Fujian, Jiangxi, and Shandong; } \\
\hline \multicolumn{5}{|c|}{ Central- south, Henan, Hubei, Hunan, Guangdong, Guangxi, and Hainan; } \\
\hline \multicolumn{5}{|c|}{ South-west, Chongqing, Sichuan, Guizhou, Yunnan, and Tibet; } \\
\hline \multicolumn{5}{|c|}{ North-west, Shann'xi, Gansu, Qinghai, Ningxia, and Sinkiang. } \\
\hline \multicolumn{5}{|c|}{$\begin{array}{l}\text { Minor,A1, D, F1, G, H, CRF02_AG, CRF03_AB, CRF06_Cpx, CRF15_01B, CRF18_Cpx, CRF24_BG, CRF33_01B, CRF55_01B, CRF57_BC, CRF58_01B, } \\
\text { CRF59_01B, CRF61_BC, CRF62_BC, CRF63_02A1, CRF64_BC, CRF65_cpx, CRF67_01B, CRF68_01B, CRF78_CPX, CRF79_0107, CRF82_Cpx, } \\
\text { CRF83_CPx, CRF85_BC, CRF86_BC, CRF87_CPx, CRF88_BC, and CRF96_cpx. }\end{array}$} \\
\hline \multicolumn{5}{|c|}{ aData are $\mathrm{n}(\%)$. } \\
\hline \multicolumn{5}{|c|}{ bUnivariable logistic regression analysis. } \\
\hline cMultivariable & analysis. & & & \\
\hline
\end{tabular}

In univariable logistic analyses, the risk of AIDSAD was significantly associated with sex, age, transmission risk group, and HIV subtype. After adjustment for these factors in the multivariable analysis, patients infected with CRF07_BC had only less than half of the risk of AIDSAD than those infected with subtype $\mathrm{B}(\mathrm{OR} 0.46,95 \% \mathrm{Cl} 0.39-0.53)$. Patients aged 45 years or older had a higher risk of AIDSAD than that of younger patients ( $<18$ years, [OR for individuals aged $45-64$ years vs. $<18$ years : $3.36,95 \% \mathrm{Cl} 1.47-8.87$; OR for $\geq 65$ vs. $<18$ years:4.32, 95\% Cl: $1.81-11.75$ ]). The risk of AIDSAD was lower in MSM than that in heterosexual patients (OR: $0.67,95 \% \mathrm{Cl} 0.6-0.75$ ).

The Yi ethnicity was associated with a lower risk of AIDSAD (OR: $0.43,95 \% \mathrm{Cl} 0.17-0.9)$; however, the sample size was very small. Two sensitivity analyses concerned only with heterosexuals and MSM were performed, and the outcomes were consistent with the whole population (data not shown).

\section{Discussion}

To our knowledge, this is the largest study to date reporting on the national distribution and trends of HIV subtypes in China, with a sample size of over 20,000, and spanning 1994-2020. ${ }^{4}$ These data showed that the HIV epidemic in China exhibited some of the greatest global genetic diversity, consisting of 38 HIV subtypes. The only other country to match China is the United States, which has approximately 15 subtypes. ${ }^{6,7}$ This high and sharp increase in HIV subtype diversity in China is consistent with evidence from most regions of the world. ${ }^{3-9}$ Although there were variations in the prevalence of the three major subtypes, the combined prevalence of these subtypes was stable throughout the study period, suggesting they might be an indicator of equally stable HIV transmission in China.

The data revealed that the previously described subtype compartmentalization ${ }^{4}$ no longer existed in the transmission risk group or was weaken in the geographic region, but persisted in the Uyghur and Yi ethnicity, which was as strong as it ever. Global travel and acquisition of infection abroad, population floating, and domestic transmission all likely contribute to increasing HIV viral diversity. ${ }^{4-9}$ 
The comparison of disease progression between subtype $B$ and other subtypes has been hindered by the fact that there are few populations with multiple circulating subtypes, including subtype B. ${ }^{15-26}$ The epidemic in China characterized by CRF01_AE, CRF07_BC, and subtype B co-circulating provided a unique opportunity for such a direct comparison. The data revealed that CRF07_BC progressed slower than subtype B, while CRF01_AE progressed as fast as subtype B.

Consistent with the results of concerted action of seroconversion to AIDS and death in Europe (CASCADE) ${ }^{16,17}$, disease progression did not differ significantly by sex. The middle (45-64 years) and the older ( $\geq 65$ years) age groups had the faster disease progression than the young ( $<18$ years). However, a lower disease progression was observed in MSM compare to that of heterosexuals.

We hypothesize that this difference could be attributed the shorter interval between seroconversion and diagnosis in MSM compare to that of heterosexuals, because most targeted HIV testing campaigns in China have always focused on the MSM population. ${ }^{2}$

The CRF07_BC strains is a relatively young HIV strain, that originated in IDU in China and is mainly confined to China. ${ }^{28}$ During the past two decades, the number of individuals infected with CRF07_BC has undergone a significant increase in China, accounting for 38\% of all infections in phase 20182020. Although it descends from the two most popular strains in the world (subtypes B and C), CRF07_BC displays many unique characteristics that differ from those of its parent strains. Li, et al. have also observed that individuals infected with CRF07_BC have a significantly higher baseline CD4 counts than those infected with CRF01_AE. ${ }^{14}$ However, they did not realize that the higher CD4 counts could be regarded as a proxy of slower disease progression, nor did they generalize from their finding the conclusion that CRF07_BC progresses slower than CRF01_AE. We have previously shown that CRF07_BC has a lower TDR prevalence than subtype B and CRF01_AE (1.5\% vs. 4.8\% vs. 5.6\%) respectively. ${ }^{29,30} \mathrm{Ge}$ et al. ${ }^{31}$ and Cao et al. ${ }^{32}$ have demonstrated that CRF07_BC is associated with better immune recovery in Chinese patients undergoing antiretroviral treatment (ART) compared to that of patients infected with CRF01_AE. Taken together, these results support the hypothesis that CRF07_BC is less pathogenic than subtype B.

Before 2014, people in China tended to accept the viewpoint that the Chinese HIV infectors will have approximately ten years AIDS-free time before they enter the AIDS phase, as report by the CASCADE study. ${ }^{16,17}$ In 2014 , Li, et al. showed that infection with CRF01_AE is associated with faster disease progression in Chinese patients infected through the sexual transmission risk group compare to that of infection with non-CRF01_AE (most were CRF07_BC and subtype B). ${ }^{10}$ The time interval between seroconversion and AIDS was only 4.8 years for CRF01_AE. The non-difference in disease progression for CRF01_AE and subtype B in these findings suggested that the time from seroconversion and AIDS for subtype B was far shorter than that previously believed. Two explanations are suggested. First, since ethnicity has proven to be a major determinant of disease progression, ${ }^{18} \mathrm{HIV}$ subtype B may progress faster in Han individuals in China than in western individuals. Second, as Wertheim, et al. have suggested, HIV subtype B is experiencing natural selection to be more virulent. ${ }^{33}$

The current study has significant implications for clinical practice and policy-making. First, since approximately $60 \%$ of patients (subtype B plus CRF01_AE accounted for 58.2\%) with new infections in China will progress to AIDS within 4.8 years, these findings justify early treatment. Second, results of this study necessitate subtype-specific monitoring and treatment guidelines. Patients with CRF07_BC may have a better prognostic treatment outcome. Third, in evaluating AIDS disease burden, the prevalence of CRF07_BC should be taken into account.

This study has several limitations. First, although it is the largest study of this kind, this study represents only approximately two percent of all individuals living with HIV in China. Thus, these findings might not be fully representative. Second, viral loads (VL) information was not included in the study, which did not permit the evaluation of the association between VL and subtype. However, this is a goal of a future study. Third, the biological mechanisms hidden behind these observations were not elucidated. At this, Huang et al. ${ }^{12}$ have shown that patients infected with CRF07_BC have significantly lower VL than that of patients infected with subtype $B$, which may be due to the deletion of seven amino acids that overlap with the apoptosis-linked gene 2-interacting protein (Alix) protein-binding domain of the p6 ${ }^{\mathrm{gag}}$. Fourth, the infection time for most of the participants was unavailable, so the rate of CD4 count decline per year could not be assessed. Indeed, as China implemented the World Health Organization (WHO)'s 'treat-all', 'treat-early', and 'treatment as prevention' policy in $2016^{34,35}$, approximately $90 \%$ of individuals with HIV were treated with ART within the first year after diagnosis, as to evaluate the natural disease progression was not only impractical, but unethical. This study provides a novel method to directly compare the rate of natural disease progression between subtypes, that is, the duration between the infection and the diagnosis as follow-up time, and to treat the follow-up time as a matching variable in multivariable logistic analysis.Fifth, the MSM population was most likely over-represented in the study sample. However, the original data, from which stratified and weighted results may be easily calculated, has been provided.

In summary, these results highlight a China HIV epidemic characterized by a high prevalence of CRF01_AE, CRF07_BC, and subtype B infections, with an overall increasing subtype diversity over the past 26 years, providing a unique opportunity to directly compare disease progression among the three subtypes.

Disease progression was slower with CRF07_BC infection than with that of subtype B infection. Moreover, for the first time, it was shown that CRF01_AE progressed as fast as subtype B. Future studies focusing on the effect of subtype on the outcome of ART, which include more confounding variables, such as VL, will help improve clinical practice and policymaking.

\section{Declarations}


The datasets used and analyzed during the current study are available from the corresponding author on reasonable request.

\section{Acknowledgements}

We thank the study participants and the staff at the collaborating clinical sites and laboratories. We thank the local health workers of the BHLN, who spent numerous hours and great effort in obtaining, verifying, and cleaning the data used in this study. We thank Professor Xiuling Zhang, from Inner Mongolia Agricultural University, for English editing the first draft of the manuscript, and Mr. Erin M, from Editage (www.editage.com) for English editing for the final draft.

\section{Authors' contributions}

$\mathrm{HL}$ and $\mathrm{YS}$ conceived and designed the study.

JY conducted and supervised the laboratory testing.

JY provided epidemiological data from the national HIV epidemiology database.

JY did the statistical analyses, made the figures and wrote the first draft.

$\mathrm{HL}$ and YS checked and supervised the statistical analyses.

All authors have reviewed the latest version of the manuscript and have approved its content.

\section{Funding}

This work was supported by Beijing Natural Science Foundation (D161100000416002).

\section{Competing interests}

The authors declare that they have no competing interests.

\section{Additional information}

Correspondence and requests for materials should be addressed to H.L. or Y.S.

\section{References}

1. National Health Commission of the People's Republic of China. Overview of notifiable infectious diseases nationwide, http://www.nhc.gov.cn/jkj/s3578/202102/2272547b05674a40a030a83eefede958.shtml. (accessed Dec 1, 2020).

2. China Center for Disease Prevention and Control. National progress in the prevention and treatment of AIDS and hepatitis $\mathrm{C}$ of China in 2020 and priority areas of work in 2021, National annual meeting on the prevention and treatment of AIDS and hepatitis C of China, Beijing China.

3. Joris Hemelaar, R. et al. Global and regional molecular epidemiology of HIV-1,19902015: a systematic review, global survey, and trend analysis. Lancet Infect Dis, 19, 143-155 (2019).

4. Xiang He, H. et al. A comprehensive mapping of HIV-1 genotypes in various risk groups and regions across China based on a nationwide molecular epidemiologic survey. PLoS One, 7, e47289 (2012).

5. The UK Collaborative Group on HIV Drug Resistance.The increasing genetic diversity of HIV-1 inthe UK, 2002-2010.AIDS 2014;28:773 - 80.

6. Richard, L., Kline, N., Saduvala, T. \& Zhang, Alexandra, M. Oster.Diversity and characterization of HIV-1 subtypes in the United States, 20082016 . Ann Epidemiol, 33, 84-88 (2019).

7. Ann, M., Dennis,Stephane Hue, C. \& Miller,Joseph, J. E. Rising prevalence of non-B HIV-1 subtypes in North Carolina and evidence for local onward transmission. Virus Evol 2017;3:vex013.

8. Stecher, M. et al. Molecular epidemiology of the HIV epidemic in three German metropolitan regions-Cologne/Bonn, Munich and Hannover, 19992016. Sci Rep, 8, 6799 (2018).

9. Alison Castley, S. et al. A national study of the molecular epidemiology of HIV-1 in Australia 20052012. PLoS One, 12, e0170601 (2017).

10. Li, Y. et al. CRF01_AE subtype is associated with X4 tropism and fast HIV progression in Chinese patients infected through sexual transmission. AIDS, 28, 521-530 (2014).

11. Hongshuo Song, W. et al. Disparate impact on CD4 T cell count by two distinct HIV-1 phylogenetic clusters from the same clade. Proc Natl Acad Sci US A, 116, 239-244 (2019).

12. Huang, S. W. et al. Patients infected with CRF07_BC have significantly lower viral loads than patients with HIV-1 subtype B: mechanism and impact on disease progression. PLoS One, 9, e114441 (2014).

13. Chu, M. et al. HIV-1 CRF01_AE strain is associated with faster HIV/AIDS progression in Jiangsu Province. China.Sci Rep, 7, 1570 (2017). 
14. Li, X. et al. HIV-1 genetic diversity and its impact on baseline CD4 + T cells and viral loads among recently infected men who have sex with men in Shanghai. China.PLoS One, 10, e0129559 (2015).

15. Pai, N. P. \& Shivkumar, S. Jorge Martinez Cajas.Does genetic diversity of HIV-1 non-B subtypes differentially impact disease progression in treatment-naive HIV-1-infected individuals? A systematic review of evidence: 19962010. J Acquir Immune Defic Syndr, 59, 382-388 (2012).

16. CASCADE Collaboration. Differences in CD4 cell counts at seroconversion and decline among 5739 HIV-1-infected individuals with well-estimated dates of seroconversion. J Acquir Immune Defic Syndr, 34, 76-83 (2003).

17. Sara Lodi,Andrew Phillips,Giota Touloumi. et al. Time from human immunodeficiency virus seroconversion to reaching CD $4+$ cell count thresholds $<200,<350$, and < 500 Cells/mm3: assessment of need following changes in treatment guidelines. Clin Infect Dis, 53, 817-825 (2011).

18. Marina, B. et al. The effects of HIV-1 subtype and ethnicity on the rate of CD 4 cell count decline in patients naive to antiretroviral therapy: a Canadian-European collaborative retrospective cohort study. CMAJ Open, 2, E318-29 (2014).

19. Giota Touloumi,Nikos Pantazis,Deenan Pillay. et al. Impact of HIV-1 subtype on CD4 count at HIV seroconversion, rate of decline, and viral load set point in European seroconverter cohorts. Clin Infect Dis, 56, 888-897 (2013).

20. Pauli, N. et al. Disease progression by infecting HIV-1 subtype in a seroconverter cohort in sub-Saharan. Africa.AIDS, 27, 2775-2786 (2013).

21. Jared, M. Baeten,Bhavna Chohan,Ludo Lavreys,et al.HIV-1 subtype D infection is associated with faster disease progression than subtype $A$ in spite of similar plasma HIV-1 loads. J Infect Dis, 195, 1177-1180 (2007).

22. Noah Kiwanuka, M. B. C. B. et al. HIV-1 viral subtype differences in the rate of CD4 + T-cell decline among HIV seroincident antiretroviral naive persons in Rakai district. Uganda.J Acquir Immune Defic Syndr, 54, 180-184 (2010).

23. Jared, M. Baeten,Bhavna Chohan,Ludo Lavreys,et al.HIV-1 subtype D infection is associated with faster disease progression than subtype A in spite of similar plasma HIV-1 loads. J Infect Dis, 195, 1177-1180 (2007).

24. Noah Kiwanuka,Oliver Laeyendecker,Merlin Robb. et al. Effect of human immunodeficiency virus Type 1 (HIV-1) subtype on disease progression in persons from Rakai, Uganda, with incident HIV-1 infection. J Infect Dis, 197, 707-713 (2008).

25. Pauli, N. et al. Disease progression by infecting HIV-1 subtype in a seroconverter cohort in sub-Saharan Africa. AIDS, 27, 2775-2786 (2013).

26. Wanna Leelawiwat, S. et al. Association between HIV genotype, viral load and disease progression in a cohort of Thai men who have sex with men with estimateddates of HIV infection. PLoS One, 13, e0201386 (2018).

27. Feng, Y. et al. The rapidly expanding CRF01_AE epidemic in China isdriven by multiple lineages of HIV-1 viruses introduced in the 1990. s.AIDS, 27 , 1793-1802 (2013).

28. Zhefeng Meng, R. et al. A new migration map of HIV-1 CRF07_BC in China: analysis of sequences from 12 provinces over a decade. PLoS One, 7, e52373 (2012).

29. Jingrong et al. Mingqiang Hao,Hui Xing, Transmitted HIV drug resistance among individuals with newly diagnosed HIV infection: a multicenter observational study. AIDS 2020;34:609 - 19.

30. Jingrong et al. Mingqiang Hao,Hui Xing, Characterization of subtypes and transmitted drug resistance strains of HIV among Beijing residents between 20012016. PLoS One 2020; 15: e0230779.

31. Zhangwen Ge, Y., Feng, K. \& Li et al.CRF01_AE and CRF01_AE cluster 4 are associated with poor immune recovery in Chinese patients under cART.Clin Infect Dis2020;ciaa380.

32. Zhiqiang Cao, J. et al. Effects of HIV-1 genotype on baseline CD4 + cell count and mortality before and after antiretroviral therapy. Sci Rep, 10, 15875 (2020).

33. Joel, O. et al. Natural selection favoring more transmissible HIV detected in United States molecular transmission network. Nat Commun, 10,5788 (2019).

34. National Health Commission of the People's Republic of China.The general office of the National Health and Family Planning Commission (NHFPC) has issued a notice concerning the adjustment of the standard of National Free Antiretroviral Treatment Programme(NFATP) for

AIDS.www.nhfpc.gov.cn/yzygj/s3593/201606/0b0fa78e10dc41328e842b1bf9cd433e.shtml.(accessed Dec 1, 2020).

35. WHO.Most countries adopt HIV policies to "treat all", http://www.who.int/hiv/mediacentre/news/treatall-policy-icasa2017/en/. (accessed Dec 1, 2020).

36. Morgan, N., Price, P. S., Dehal, Adam, P. \& Arkin FastTree 2-approximatelymaximum-likelihood trees for large alignments. PLoS One, 5, e9490 (2010).

37. Daniel Struck, G. et al. COMET: adaptive context-based modeling for ultrafast HIV-1 subtype identification. Nucleic Acids Res, 42, e144 (2014).

\section{Figures}


13,230 sequences submitted by Beijing HIV laboratory network for resistance genotyping from newly diagnosed individual between 2001 and 2020 in China
7,433 sequences with known sampling province,sampling year, and transmission risk sampled in China in HIV Los Alamos database

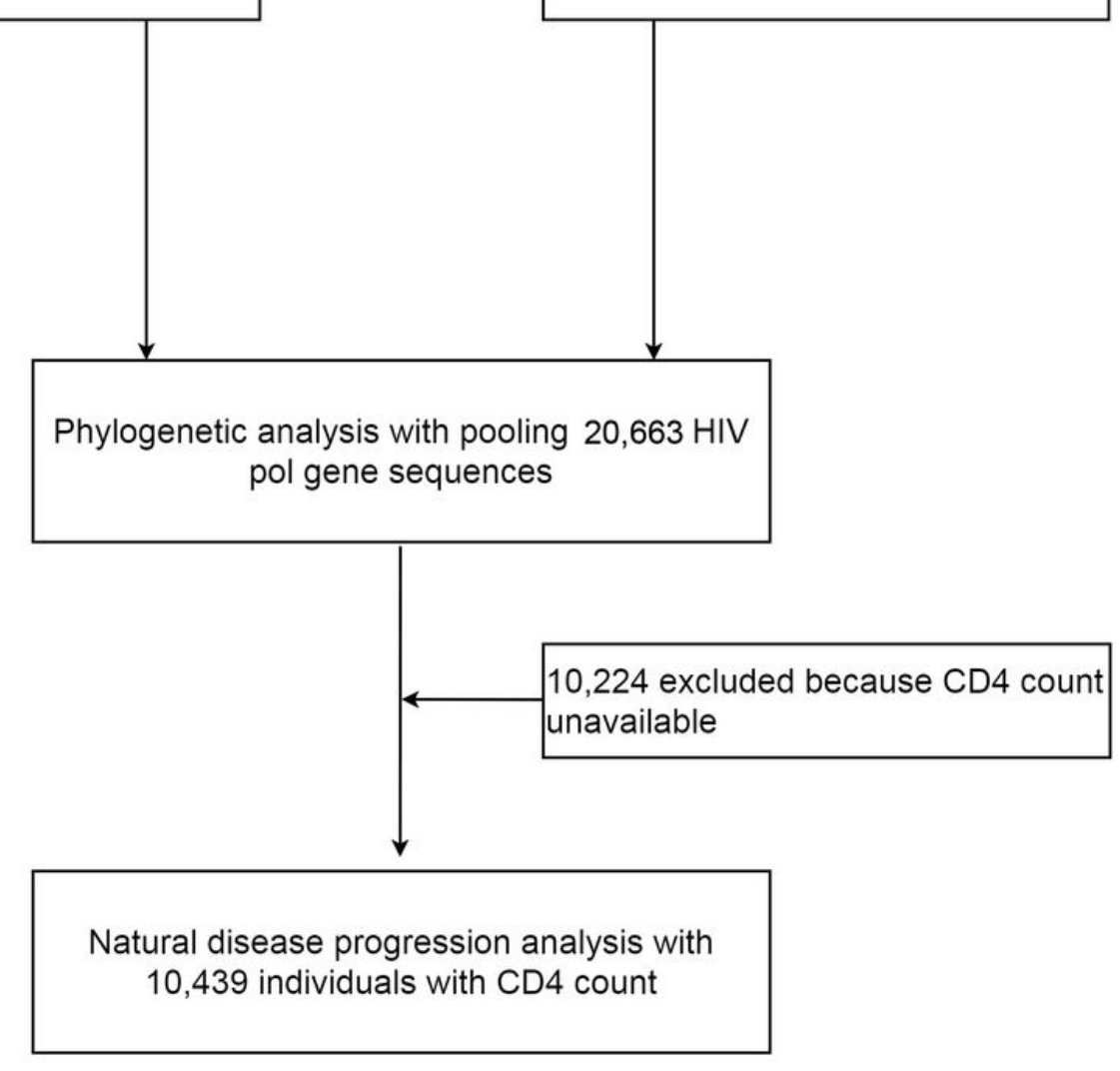

Figure 1

Study profile. 


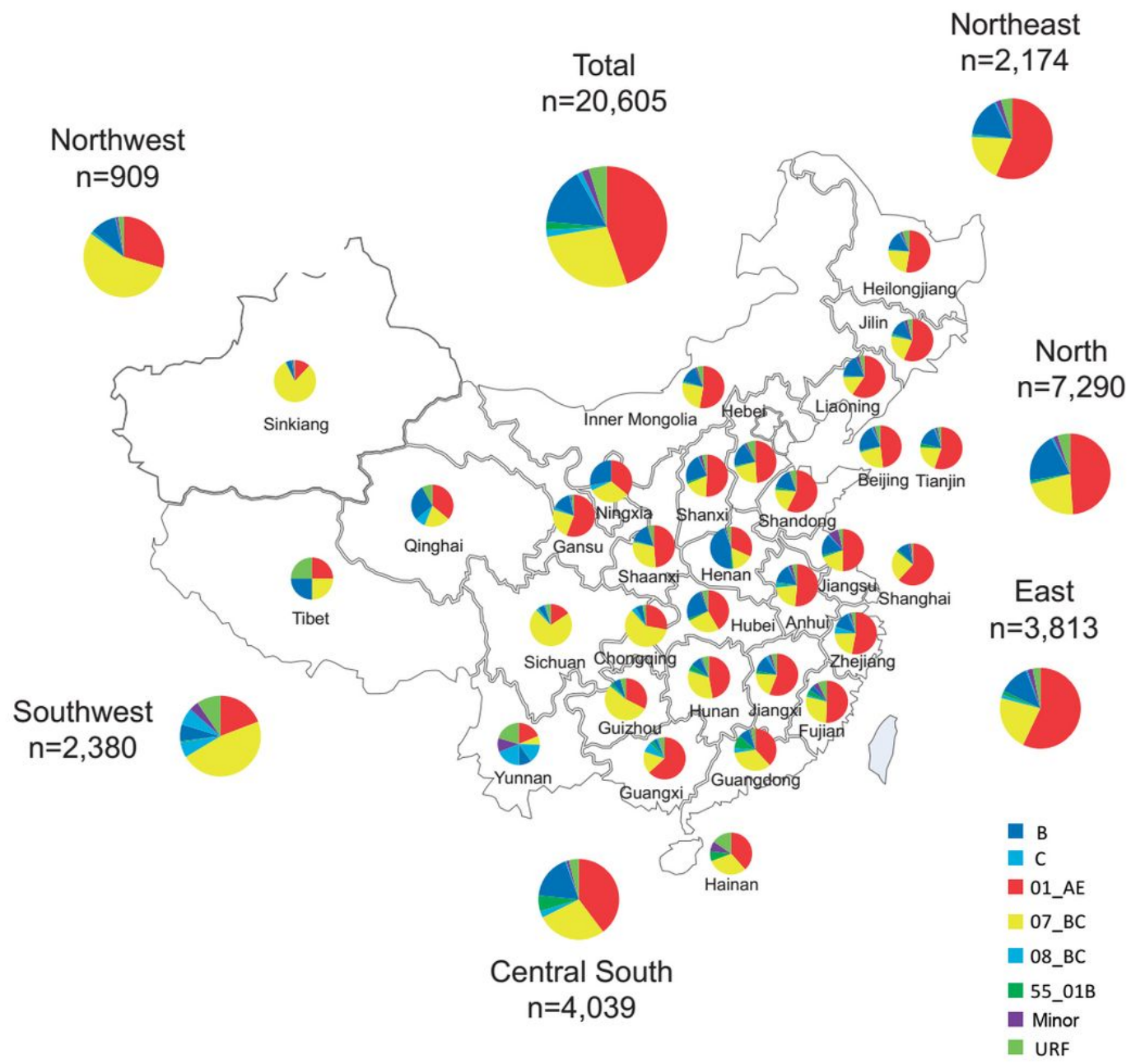

Figure 2

Geographical distribution of HIV subtype. Seven common subtypes, CRF01_AE, CRF07_BC, subtype B, URF, CRF55_01B, CRF08_BC, and subtype C. Samples were from 31 provinces of China. North, Beijing, Tianjin, Hebei, Shanxi, and Inner Mongolia; Northeast, Liaoning, Jilin, and Heilongjiang; East, Shanghai, Jiangsu, Zhejiang, Anhui, Fujian, Jiangxi, and Shandong; Central-south, Henan, Hubei, Hunan, Guangdong, Guangxi, and Hainan; Southwest, Chongqing, Sichuan, Guizhou, Yunnan, and Tibet; Northwest, Shann'xi, Gansu, Qinghai, Ningxia, and Sinkiang. 


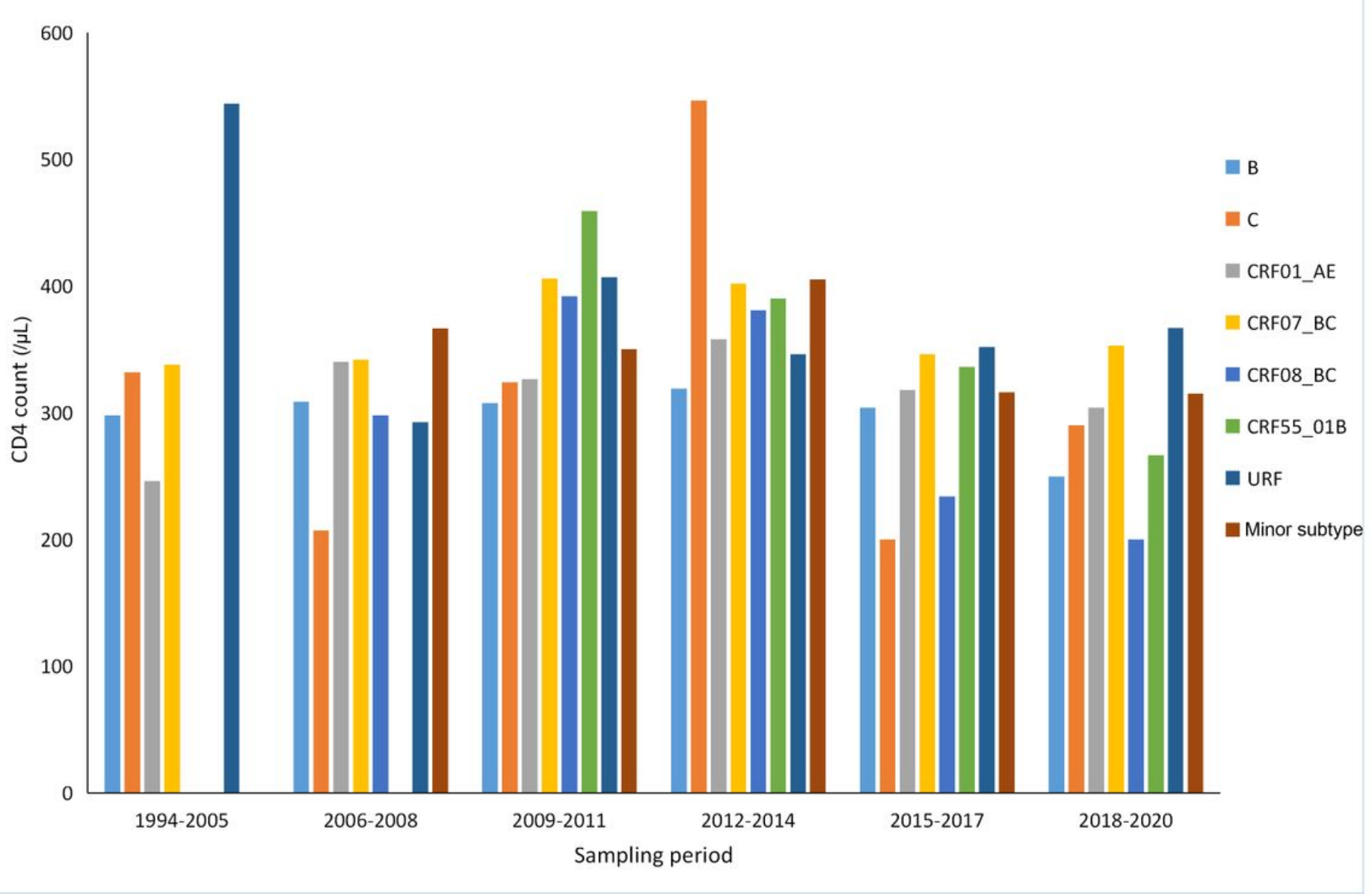

Figure 3

The CD4 count of the seven common subtypes.

\section{Supplementary Files}

This is a list of supplementary files associated with this preprint. Click to download.

- 07BCFigureSlegends.docx

- 07BCtables1.pdf

- 07BCtables2.pdf

- 07FigS1.pdf

- 07FigS2.pdf

- 07FigS3.pdf 